\title{
Rotating charged hairy black hole in $(2+1)$ dimensions and particle acceleration
}

\author{
J. Sadeghi*,B. Pourhassan † and H. Farahani ${ }^{\ddagger}$ \\ Young researchers club, Ayatollah Amoli branch, Islamic azad university, Amol, Iran
}

June 29, 2018

\begin{abstract}
In this paper we construct rotating charged hairy black hole in $(2+1)$ dimensions for infinitesimal black hole charge and rotation parameters. Then we consider this black hole as particle accelerator and calculate the center-of-mass energy of two colliding test particles near the rotating charged hairy black hole in $(2+1)$ dimensions. As we expected, the center-of-mass energy has infinite value.
\end{abstract}

Keywords: Particle Acceleration; 3D Black Hole; Thermodynamics.

\section{Introduction}

Recently, charged black hole with a scalar hair in $(2+1)$ dimensions studied in the Ref. [1] and rotating hairy black hole in $(2+1)$ dimensions studied in the Ref. [2]. The $(2+1)$ dimensional theories are toy models to investigate some fundamental ideas to understand higher dimensional theories because they are easy to study [3]. Also, it is useful to study gauge/gravity dualities [4-7]. These kinds of black hole recover well known BTZ black hole in $(2+1)$ dimensions [8-12].

In this work we would like collect Refs. [1, 2] to construct rotating charged hairy black hole in $(2+1)$ dimensions. In that case we assume that the electric charge and rotational parameter are infinitesimal.

Then, the main goal of this paper is particle acceleration mechanism. It has been shown that free particles falling from rest at infinity outside a rotating black holes may collide with arbitrarily high center-of-mass (CM) energy and hence rotating black holes may be considered as a particle accelerator $[13,14]$. It is found that the CM energy of elastic and inelastic scattering of particles in the gravitational field of static and rotating Kerr black

\footnotetext{
*Email: pouriya@ipm.ir

${ }^{\dagger}$ Email: b.pourhassan@umz.ac.ir

${ }^{\ddagger}$ Email: h.farahani@umz.ac.ir
} 
holes is limited for the static and is unlimited for the rotating black holes [15]. Several studies indicate that having infinite CM energy of colliding particles is a generic property of a rotating black holes [16-27]. Now, we verify this general property for the rotating charged hairy black hole in $(2+1)$ dimensions.

In the next section we construct a black hole in $(2+1)$ dimensions include electric charge, scalar charge and rotational parameter, and then in section 3 we obtain field equations and discuss geometric properties of this black hole. In section 4 we discuss about horizon structure of rotating charged hairy black hole and obtain event horizon for small radius limit. In section 5 we study particle acceleration and obtain CM energy of two colliding particles near the black hole. In section 6 we discuss about the effective potential, and finally in section 7 we summarized our results and give conclusion.

\section{Rotating charged hairy black hole in $(2+1)$ dimen- sions}

We consider the $(2+1)$-dimensional gravity with a non-minimally coupled scalar field and self coupling potential $V(\phi)$, which is described by the following action,

$$
S=\frac{1}{2} \int d^{3} x \sqrt{-g}\left[R-g^{\mu \nu} \nabla_{\mu} \phi \nabla_{\nu} \phi-\xi R \phi^{2}-2 V(\phi)-\frac{1}{4} F_{\mu \nu} F^{\mu \nu}\right],
$$

where $\xi$ is a coupling constant between gravity and the scalar field which will be fixed as $\xi=1 / 8$. The proposed metric for this black hole is similar to the Ref. [2],

$$
d s^{2}=-f(r) d t^{2}+\frac{1}{f(r)} d r^{2}+r^{2}(d \psi+\omega(r) d t)^{2} .
$$

Under assumption of infinitesimal $a$ and $Q$ we obtain,

$$
f(r)=3 \beta-\frac{Q^{2}}{4}+\left(2 \beta-\frac{Q^{2}}{9}\right) \frac{B}{r}-Q^{2}\left(\frac{1}{2}+\frac{B}{3 r}\right) \ln (r)+\frac{(3 r+2 B)^{2} a^{2}}{r^{4}}+\frac{r^{2}}{l^{2}}+\mathcal{O}\left(a^{2} Q^{2}\right) .
$$

$Q$ is the electric charge, $a$ is a rotation parameter and is related to the angular momentum

of the solution and $l$ is related to the cosmological constant via $\Lambda=-\frac{1}{l^{2}}$. $\beta$ is integration constants depends on the black hole charge and mass,

$$
\beta=\frac{1}{3}\left(\frac{Q^{2}}{4}-M\right)
$$

and scalar charge $B$ related to the scalar field as,

$$
\phi(r)= \pm \sqrt{\frac{8 B}{r+B}} .
$$

Also one can obtain,

$$
\omega(r)=-\frac{(3 r+2 B) a}{r^{3}}
$$


and,

$$
V(\phi)=\frac{2}{l^{2}}+\frac{1}{512}\left[\frac{1}{l^{2}}+\frac{\beta}{B^{2}}+\frac{Q^{2}}{9 B^{2}}\left(1-\frac{3}{2} \ln \left(\frac{8 B}{\phi^{2}}\right)\right)\right] \phi^{6}+\mathcal{O}\left(Q^{2} a^{2} \phi^{8}\right) .
$$

We can see that the first order of rotational parameter $a$ has no effect on potential. So, this potential studied completely in the Ref. [1].

In the next section we write field equations and discuss geometric properties of solution briefly.

\section{$3 \quad$ Field equations}

One can obtain the following independent Christophel symbols,

$$
\begin{aligned}
\Gamma_{t t}^{r} & =-\Gamma_{t r}^{t}=\frac{36 r^{3}-9 l^{2} Q^{2} r+6 B l^{2} Q^{2} \ln (r)-4 B l^{2} Q^{2}-36 \beta B l^{2}}{36 l^{2} r^{2}} \\
\Gamma_{t r}^{\psi} & =\Gamma_{r \psi}^{t}=-\Gamma_{t \psi}^{r}=\frac{a B}{r^{2}} \\
\Gamma_{r \psi}^{\psi} & =-\Gamma_{\psi \psi}^{r}=r \\
\Gamma_{r r}^{r} & =\frac{\mathcal{A}}{\mathcal{B}^{2}}
\end{aligned}
$$

where,

$$
\begin{aligned}
\frac{\mathcal{A}}{36 l^{2} r^{3}} & =-36 r^{6}+9 l^{2} Q^{2} r^{4}+4 B l^{2}\left(9 \beta+Q^{2}\right) r^{3}+324 l^{2} a^{2} r^{2}+648 B l^{2} a^{2} r \\
& +288 l^{2} a^{2} B^{2}-6 B l^{2} Q^{2} \ln (r) r^{3} \\
\mathcal{B} & =36 r^{6}+9 l^{2}\left(12 \beta-Q^{2}\right) r^{4}+4 B l^{2}\left(18 \beta-Q^{2}\right) r^{3}+324 l^{2} a^{2} r^{2}+432 B l^{2} a^{2} r \\
& +144 l^{2} a^{2} B^{2}-12 B l^{2} Q^{2} \ln (r) r^{3}-18 l^{2} Q^{2} \ln (r) r^{4} .
\end{aligned}
$$

These yield to the following Ricci scalar,

$$
R=-\frac{36 r^{6}-3 l^{2} Q^{2} r^{4}+2 B l^{2} Q^{2} r^{3}+216 B l^{2} a^{2} r+180 l^{2} a^{2} B^{2}}{6 l^{2} r^{6}},
$$

which is singular at $r=0$, and yields to $R=-\frac{6}{l^{2}}$ for $Q=0$ and $a=0$. The Riemann and Ricci tensors also are singular at $r=0$ for $Q \neq 0$ and $a \neq 0$.

One of the non-vanishing components of Cotton tensor obtained as the following,

$$
C_{\psi r \psi}=\left(\frac{3 B}{4 r^{2}}-\frac{B \ln (r)}{2 r^{2}}+\frac{1}{4 r}\right) Q^{2}+\left(\frac{54}{r^{3}}+\frac{180 B}{r^{4}}+\frac{120 B^{2}}{r^{5}}\right) a^{2}+\frac{3 \beta B}{r^{2}},
$$

which tells that, at the non conformal limit, the metric is flat. Therefore, the rotating charged hairy black holes are geometrically quite different from the case of the static uncharged BTZ black hole. 


\section{Horizon structure}

In the Refs. [1] and [2] horizon structure of hairy $(2+1)$ dimensional black holes in several special cases such as uncharged, extremal rotating and non-extremal rotating black holes, and also under some constraint on the black hole parameters has been studied in details. Now we would like to discuss a more general cases $(Q \neq 0, a \neq 0, B \neq 0)$ without any conditions on the black hole parameters. Just we take $r=z+1$ and assume $Z$ as infinitesimal parameter $(z \ll 1$ or $r-1 \ll 1)$, therefore $\ln (r) \approx r-1$. Under this assumption, the relation (3) gives the following equation,

$$
\begin{aligned}
x=r^{4} f(r) & =r^{6}-\frac{Q^{2}}{2} r^{5}+\left(\frac{Q^{2}}{2}-\frac{B Q^{2}}{3}-M\right) r^{4} \\
& +\frac{B}{3}\left(\frac{7}{6} Q^{2}-2 M\right) r^{3}+9 a^{2} r^{2}+12 B a^{2} r+4 B^{2} a^{2}=0 .
\end{aligned}
$$

The equation (12) has generally six solutions. Two of them are imaginary and are not physically interesting cases. Also two of them are negative and interpreted as naked singularities. Two remaining solutions which are real positive are indeed inner and outer horizons. In order to find event horizon we decompose equation (12) as the following,

$$
x=\left(r^{2}+c_{1}\right)\left(r^{2}-c_{2}\right)\left(r+c_{3}\right)\left(r-c_{4}\right),
$$

where $r= \pm i \sqrt{c_{1}}$ are imaginary, $r=-\sqrt{c_{2}}$ and $r=-c_{3}$ are negative solutions. Only physical solutions are $r_{+}=\sqrt{c_{2}}$ and $r_{-}=c_{4}$ which will be interpreted as outer and inner horizons respectively and obtained as the following,

$$
c_{2}=\frac{B}{3 Q^{2}}\left(\frac{7}{6} Q^{2}-2 M\right)\left(-1+\sqrt{1+\frac{216 a^{2} Q^{2}}{B\left(\frac{7}{6} Q^{2}-2 M\right)^{2}}}\right),
$$

and,

$$
c_{4}=\frac{Q^{2}}{4}\left(-1+\sqrt{1+\frac{8 B}{3 Q^{2}}}\right) .
$$

It is clear that the $7 Q^{2} \geq 12 M$ is crucial condition. Typical behavior of real solutions illustrated in the Fig. 1 where $r=2$ denotes outer horizon. If we choose $Q^{2}=\frac{12}{7} M$, then $r=0$ is event horizon. 


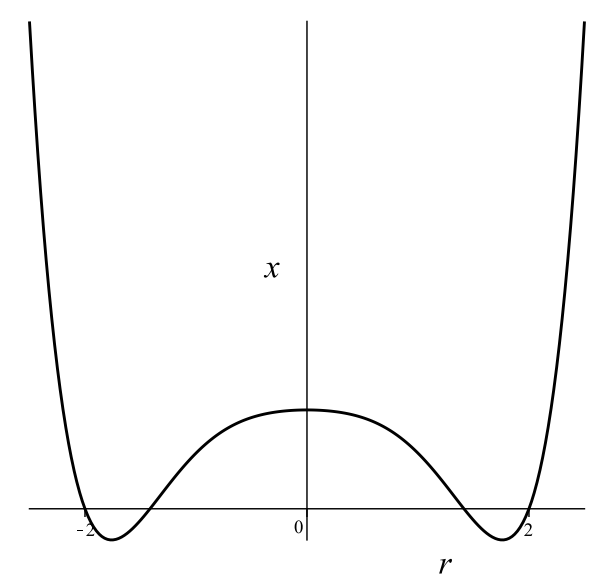

Figure 1: Horizon structure

\section{$5 \quad$ Particle acceleration}

In order to obtain the CM energy, we should calculate the velocities of the particles, which will be obtained as the following,

$$
\begin{aligned}
\dot{t} & =\frac{\omega(r) L-E}{f(r)}, \\
\dot{r} & =\sqrt{f(r)\left(1+\frac{E^{2}}{f(r)}+\frac{\omega(r)^{2} r^{2}-f(r)}{f(r) r^{2}} L^{2}-\frac{2 \omega(r) E L}{f(r)}\right)}, \\
\dot{\phi} & =\frac{\omega(r)(\omega(r) L-E) r^{2}-f(r) L}{f(r) r^{2}} .
\end{aligned}
$$

where $E$ denotes the test particle energy per unit mass and $L$ denotes the angular momentum per unit mass. We use velocity components (16) to obtain CM energy of the two-particle collision in the background rotating charged hairy $(2+1)$ dimensional black hole. It is assumed that two particles have the angular momentum per unit mass $L_{1}$ and $L_{2}$, energy per unit mass $E_{1}$ and $E_{2}$. Moreover we take $m_{0}$ as the rest mass of both particles. Then, by using the following relation,

$$
E_{C M}=\sqrt{2} m_{0} \sqrt{1+g_{\mu \nu} v_{1}^{\mu} v_{2}^{\nu}}
$$

where $v_{i}=\left(\dot{t}_{i}, \dot{r}_{i}, \dot{\phi}_{i}\right)$, we can find the CM energy of two-particle collision as the following expression,

$$
\bar{E}_{C M}=\frac{1}{f(r) r^{2}}\left(f(r) r^{2}+E_{1} E_{2} r^{2}-L_{1} L_{2}\left(f(r)-\omega(r)^{2} r^{2}\right)-\omega(r) r^{2}\left(E_{1} L_{2}+E_{2} L_{1}\right)-H_{1} H_{2}\right),
$$


where $\bar{E}_{C M} \equiv \frac{E_{C M}^{2}}{2 m_{0}^{2}}$, and,

$$
H_{i}=\sqrt{f(r) r^{2}+E_{i}^{2} r^{2}-\left(f(r)-\omega(r)^{2} r^{2}\right) L_{i}^{2}-2 \omega(r) r^{2} E_{i} L_{i}},
$$

with $i=1,2$. Using relations (3) and (6) in the equation (18) we draw typical behavior of CM energy in the Fig. 2 which shows that near the black hole horizon the CM energy takes large value which is expected for the rotational black hole. Also dotted line of the Fig. 2, which is corresponding to static black hole $(a=0)$ shows that CM energy has finite value which is also expected.

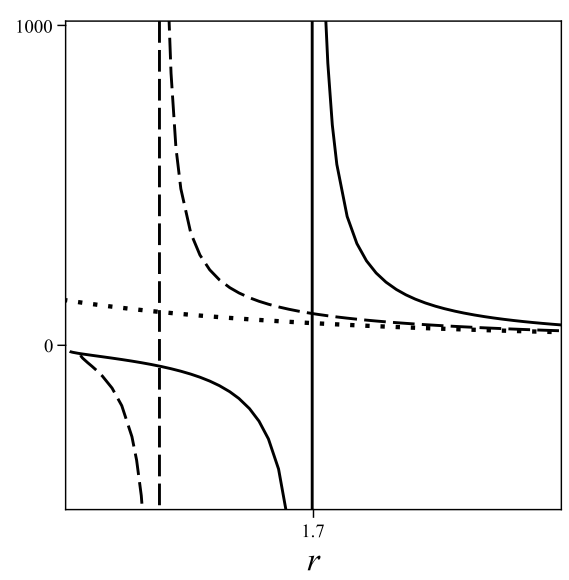

Figure 2: Plots of $\bar{E}_{C M}$ in terms of $r$ by choosing $E_{1}=E_{2}=10, L_{1}=-L_{2}=-10$, and $l=1$. We fix black hole parameters as $M=5, Q=5, B=5$ and $a=1$ (solid line), $M=1$, $Q=10, B=3$ and $a=1$ (dashed line), and $M=1, Q=10, B=3$ and $a=0$ (dotted line).

Also we can obtain $\bar{E}_{C M}$ near the horizon analytically. In that case we should expand $\bar{E}_{C M}$ for $r \rightarrow r_{+}$to obtain,

$$
\bar{E}_{C M}=\frac{r_{+}^{2}\left(\frac{\left(3 r_{+}+2 B\right)\left(L_{1}+L_{2}\right) a}{r_{+}^{3}}+E_{1}+E_{2}\right)^{2}-\left(L_{1} E_{2}-E_{1} L_{2}\right)^{2}}{2 r_{+}^{2}\left(\frac{\left(3 r_{+}+2 B\right) L_{1} a}{r_{+}^{3}}-E_{1}\right)\left(\frac{\left(3 r_{+}+2 B\right) L_{2} a}{r_{+}^{3}}-E_{2}\right)} .
$$

It yields to a critical angular momentum where CM energy will be infinite,

$$
L_{c i}=\frac{E_{i} r_{+}^{3}}{\left(3 r_{+}+2 B\right) a} \text {. }
$$

It means that the particles with the critical angular momentum $L_{c i}$ can collide with arbitrary high CM energy near the horizon. in the special case of vanishing scalar charge $(B=0)$ we can see that $L_{c i} \propto r_{+}^{2}$, which agrees with the results of [26, 27]. In the Fig. 3 we draw critical angular momentum in terms of rotational parameter $a$. 


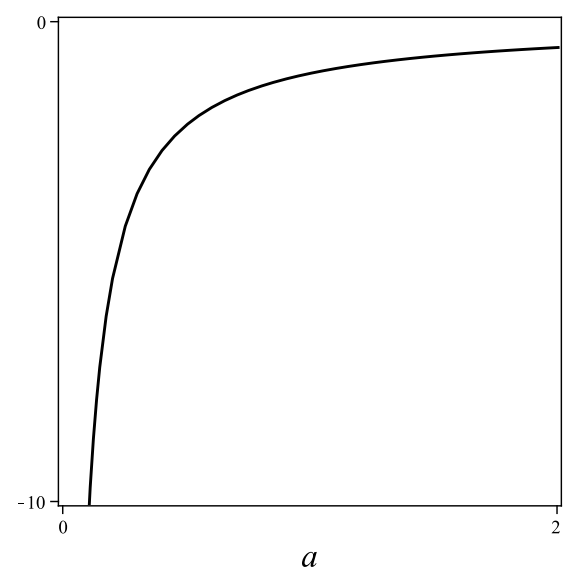

Figure 3: Plots of critical angular momentum $L_{c i}$ in terms of $a$ by choosing $E_{i}=10, M=5$, $Q=5, B=5$ and $l=1$.

\section{Effective potential}

The effective potential is given by the following relation,

$$
V_{e f f}=\frac{E^{2}-R}{2}
$$

where $R=\dot{r}^{2}$ obtained by using the relation (16). In the Fig. 4 we draw effective potential (22) for various values of the black hole parameters. Fig. 4 (a) and (b) show that there is a critical radius $r_{c}$ so for the $r>r_{c}$ electric charge $Q$ and scalar charge $B$ decreased the effective potential but for the $r<r_{c}$ the effective potential takes infinite value. Indeed, this region is near the black hole horizon and infinite value of the energy is expectable. In the special case of $r=r_{c}$ variation of $Q$ and $B$ have not any effects on the effective potential. In the Fig. 4 (c) we learn that the rotational parameter $a$ reduced the effective potential. The parameter $R$ is also useful for another reason. The particle with the critical angular momentum may have an orbit outside the outer horizon if,

$$
O=\left.\frac{d R}{d r}\right|_{r=r_{+}}>0
$$

In the Fig. 5 we draw $O$ in terms of the black hole parameters. Fig. 5 (a) tells that for the case of $a=1$ and $B=2$ the electric charge restricted as $Q \geq 5$. Fig. 5 (b) tells that for the case of $a=1$ and $Q=5$ the scalar charge restricted as $1.5<B<26$. Fig. 5 (c) tells that for the case of $Q=5$ and $B=2$ the rotational parameter restricted as $a \leq 1$. In summary we conclude that the condition of having an orbit outside the outer horizon is choosing small $a$ and large $Q$ with $B_{\min }<B<B_{\max }$, which values of $B_{\min }$ and $B_{\max }$ are depend on values of $Q$ and $a$. In another word the electric charge increases $O$ but rotational parameter decreases $O$, while scalar charge may increases or decreases $O$. 

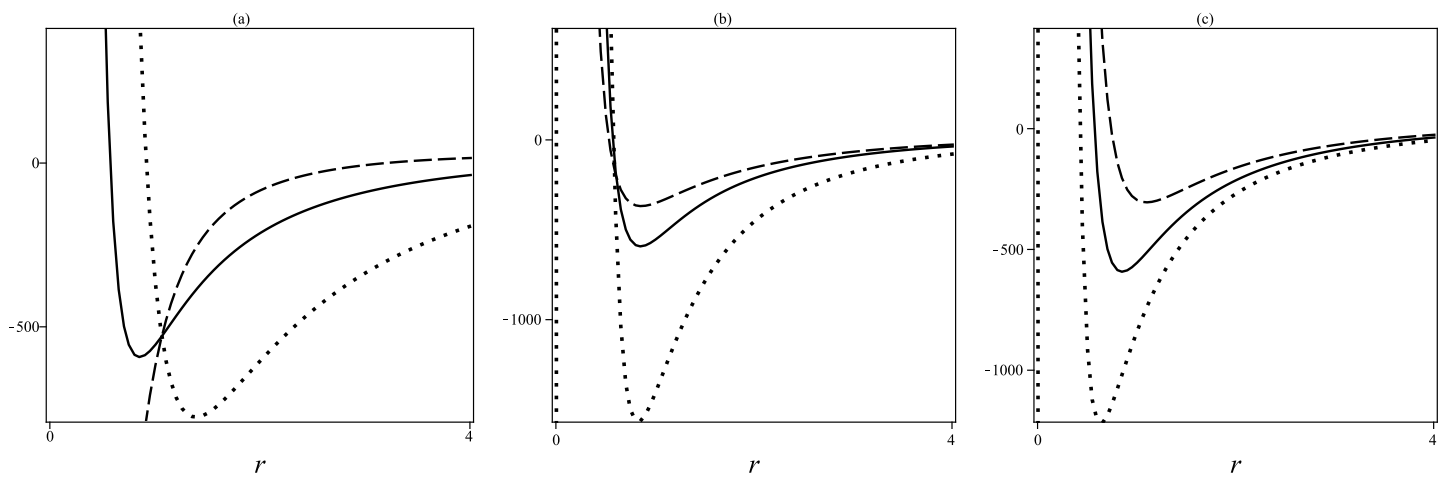

Figure 4: Plot of Effective potential in terms of $r$ for $E=5, L=10, M=5$ and $l=1$. (a) $B=1, a=1, Q=0$ (dashed line), $Q=5$ (solid line) and $Q=10$ (dashed line). (b) $Q=5$, $a=1, B=0$ (dashed line), $B=1$ (solid line) and $B=5$ (dashed line). (c) $Q=5, B=1$, $a=0$ (dashed line), $a=1$ (solid line) and $a=2$ (dashed line).
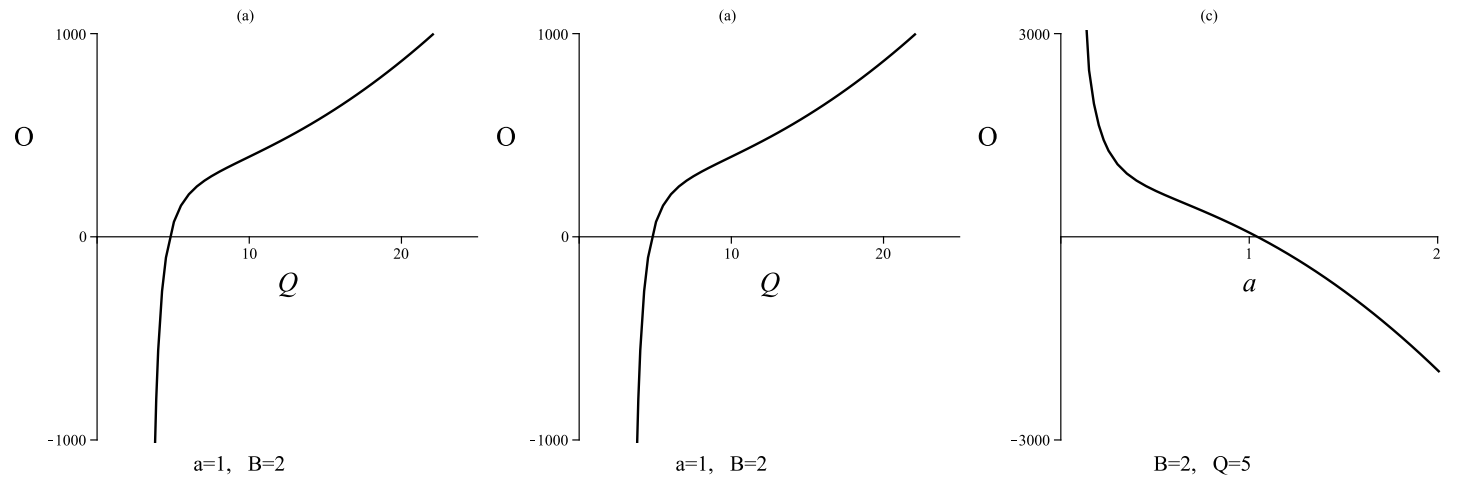

Figure 5: Plot of $O$ in terms of (a) $Q$, (b) $B$ and (c) $a$ for $E=10, M=5$ and $l=1$.

Particles on a circle orbit have the following angular momentum,

$$
\begin{aligned}
& L_{c o 1}=\frac{\omega(r) E r^{2}+r \sqrt{f(r)} \sqrt{E^{2}+\omega(r)^{2} r^{2}-f(r)}}{\omega(r)^{2} r^{2}-f(r)}, \\
& L_{c o 2}=\frac{\omega(r) E r^{2}-r \sqrt{f(r)} \sqrt{E^{2}+\omega(r)^{2} r^{2}-f(r)}}{\omega(r)^{2} r^{2}-f(r)} .
\end{aligned}
$$

It is easy to check that at $r=r_{+}$where $f\left(r_{+}\right)=0$ we have $L_{c i}=L_{c o i}$. This is also happen if $E^{2} \geq f(r)-\omega(r)^{2} r^{2}$. In these cases there is no circle orbit. Existing the circle orbit needs the angular momentum be in the interval $L \in\left[L_{c o 2}, L_{c o 1}\right]$. If we set $L_{c o 1}=L_{1}$, and $L_{c o 2}=L_{2}-\delta$, where $0 \leq \delta \leq L_{c o 1}-L_{c o 2}$, then the CM energy in the circle orbit obtained 
as the following,

$$
\begin{aligned}
\bar{E}_{C M o} & =1+\frac{E_{1} E_{2}+\sqrt{\left(E_{1}^{2}+\omega(r)^{2} r^{2}-f(r)\right)\left(E_{2}^{2}+\omega(r)^{2} r^{2}-f(r)\right)}}{\left(\omega(r)^{2} r^{2}-f(r)\right)} \\
& -\frac{\sqrt{E_{1}^{2}+\omega(r)^{2} r^{2}-f(r)}}{\sqrt{f(r)} r} \delta+\mathcal{O}\left(\delta^{2}\right) .
\end{aligned}
$$

It means that the first particle is a target and the second one on the circle orbit collide with the target. $\delta$ is the small parameter and interpreted as the drift of the second particle from the circle orbit.

\section{Conclusions}

In this work we constructed rotating charged black hole in $(2+1)$ dimensions with an scalar hair and extended recent works of Xu et al. [1,2]. We obtained event horizon for infinitesimal black hole parameters and found that electric charge and scalar charge increases size of event horizon which is agree with the results of the Ref. [1].

The main part of our paper is consideration of rotating charged hairy black hole in $(2+1)$ dimensions as particle accelerator. We confirmed that having arbitrary high CM energy of two colliding test particle near the rotating black holes is universal property. Also we found that CM energy will be finite for static black holes as expected. We found that the angular momentum corresponding to collision near the black hole is negative and decreased by rotational parameter $a$. We also discussed about effective potential and circle orbit near the black hole and found effect of black hole parameters.

There are also several open problems related to these new solutions. In the Refs. [28, 29] thermodynamics of charged and rotating hairy black holes studied separately. Now, it is interesting to have similar studies to the Refs. [30-32] for present background and investigate statistical and thermodynamical quantities.

It is interesting to obtain quasi-normal modes [33, 34] of rotating charged hairy black hole in $(2+1)$ dimensions.

As we mentioned in the introduction there are some papers which studied AdS/CFT correspondence in 3D hairy black hole. If this is the case and there is a dual CFT for this background, then an important problem will be calculation of some parameters such as drag force and jet-quenching [35-40].

\section{References}

[1] W. Xu, L. Zhao, "Charged black hole with a scalar hair in $(2+1)$ dimensions", Phys. Rev. D87 (2013) 124008

[2] L. Zhao, W. Xu, B. Zhu, "Novel rotating hairy black hole in (2+1)-dimensions", arXiv:1305.6001 [gr-qc]] 
[3] M. Hortacsu, H.T. Ozcelik, B. Yapiskan, "Properties of Solutions in 2+1 Dimensions", Gen. Rel. Grav. 35 (2003) 1209

[4] M. Henneaux, C. Martinez, R. Troncoso, and J. Zanelli, "Black holes and asymptotics of $2+1$ gravity coupled to a scalar field", Phys.Rev. D65 (2002) 104007

[5] M. Hasanpour, F. Loran, and H. Razaghian, "Gravity/CFT correspondence for three dimensional Einstein gravity with a conformal scalar field", Nucl. Phys. B867 (2013) 483

[6] D.F. Zeng, "An Exact Hairy Black Hole Solution for AdS/CFT Superconductors", arXiv:0903.2620 [hep-th]].

[7] B. Chen, Z. Xue, and J. ju Zhang, "Note on Thermodynamic Method of Black Hole/CFT Correspondence", JHEP 1303 (2013) 102

[8] M. Banados, C. Teitelboim and J. Zanelli, "The black hole in three-dimensional spacetime", Phys. Rev. Lett. 69, 1849 (1992)

[9] J. Sadeghi, H. Farahani, B. Pourhassan and M. Noorbakhsh, " Cosmic String in the BTZ Black Hole Background with Time-Dependant Tension", Physics Letters B 703 (2011) 14, arXiv:0903.0292 [hep-th]]

[10] G. Clement, "Classical solutions in three-dimensional Einstein-Maxwell cosmological gravity", Class. Quantum Grav. 10 (1993) L49

[11] G. Clement, "Spinning charged BTZ black holes and self-dual particle-like solutions", Phys. Lett. B367 (1996) 70

[12] C. Martinez, C. Teitelboim, and J. Zanelli, "Charged rotating black hole in three spacetime dimensions", Phys.Rev. D61 (2000) 104013

[13] M. Banados, J. Silk and S. M. West, "Kerr Black Holes as Particle Accelerators to Arbitrarily High Energy", Phys. Rev. Lett. 103 (2009) 111102

[14] T. Jacobson, T.P. Sotiriou, "Spinning Black Holes as Particle Accelerators", Phys. Rev. Lett. 104 (2010) 021101

[15] K. Lake, "Particle Accelerators inside Spinning Black Holes", Phys.Rev.Lett.104 (2010) 211102

[16] A. A. Grib, Yu. V. Pavlov "On particles collisions near rotating black holes", Grav. Cosmol. 17 (2011) 42

[17] S-W. Wei, Y-X. Liu, H. Guo, C-E. Fu, "Charged Spinning Black Holes as Particle Accelerators", Phys. Rev. D82 (2010) 103005 
[18] O.B. Zaslavskii, "Acceleration of particles by nonrotating charged black holes", JETP Letters 92 (2010) 571

[19] O. B. Zaslavskii, "Acceleration of particles as universal property of rotating black holes", Phys. Rev. D82 (2010) 083004

[20] O. B. Zaslavskii, "Acceleration of particles by black holes - general explanation", Class. Quant. Grav. 28 (2011) 105010

[21] M. Kimura, K. Nakao, H. Tagoshi, "Acceleration of colliding shells around a black holeValidity of test particle approximation in BSW process", Phys. Rev. D83 (2011) 044013

[22] M. Banados, B. Hassanain, J. Silk, S.M. West, "Emergent Flux from Particle Collisions Near a Kerr Black Hole", Phys. Rev. D83 (2011) 023004

[23] T. Harada, M. Kimura, "Collision of an innermost stable circular orbit particle around a Kerr black hole", Phys. Rev. D83 (2011) 024002

[24] S-W. Wei, Y-X. Liu, H-T. Li, F-W. Chen, "Particle Collisions on Stringy Black Hole Background", JHEP 1012 (2010) 066

[25] J. Sadeghi and B. Pourhassan, "Particle acceleration in Horava-Lifshitz black Holes", Eur. Phys. J. C 72 (2012) 1984 [arXiv:1108.4530 [hep-th]]

[26] Yang Li, Jie Yang, Yun-Liang Li, Shao-Wen Wei, Yu-Xiao Liu, "Particle Acceleration in Kerr-(anti-) de Sitter Black Hole Backgrounds", Class. Quantum Grav. 28 (2011) 225006

[27] C. Liu, S. Chen, C. Ding, and J. Jing, "Kerr-Taub-NUT black hole as Particle Accelerators", Phys. Lett. B701 (2011) 285

[28] A. Belhaj, M. Chabab, H. EL Moumni, M. B. Sedra, "Critical Behaviors of 3D Black Holes with a Scalar Hair", [arXiv:1306.2518 [hep-th]]

[29] J. Sadeghi, H. Farahani, "Thermodynamics of a charged hairy black hole in $(2+1)$ dimensions", arXiv:1308.1054 [hep-th]]

[30] J. Sadeghi, K. Jafarzade, and B. Pourhassan, "Thermodynamical Quantities of HoravaLifshitz Black Hole", Int. J. Theor. Phys. 51 (2012) 3891

[31] A. Pourdarvish, J. Sadeghi, H. Farahani, and B. Pourhassan, "Thermodynamics and Statistics of Godel Black Hole with Logarithmic Correction", Int. J. Theor. Phys. DOI: 10.1007/s10773-013-1658-4

[32] A. Pourdarvish and B. Pourhassan "Statistical mechanics of a new regular black hole", Int. J. Theor. Phys. DOI: 10.1007/s10773-013-1701-5 
[33] J. Sadeghi, A. Chatrabhuti and B. Pourhassan, "Quasi-normal Modes of $A d S_{5}$ Black Hole at N=2 Supergravity", International Journal of Theoretical Physics 50 (2011) 129

[34] J. Sadeghi and B. Pourhassan, K. Jafarzadeh, E. Reisi and M. Rostami, "Massless Fermion Quasinormal Modes in the Horava-Lifshitz Background", Can. J. Phys. 91(3) (2013) 251

[35] J. Sadeghi and B. Pourhassan, "Drag Force Of Moving Quark At N= 2 Supergravity", JHEP12(2008)026, arXiv:0809.2668 [hep-th]]

[36] J. Sadeghi, M. R. Setare, B. Pourhassan and S. Heshmatian, "Drag Force of Moving Quark in STU Background", Eur. Phys. J. C 61 (2009) 527 [arXiv:0901.0217 [hep-th]]

[37] J. Sadeghi, M. R. Setare and B. Pourhassan, "Drag force with different charges in STU background and AdS/CFT", J. Phys. G: Nucl. Part. Phys. 36 (2009) 115005 arXiv:0905.1466 [hep-th]]

[38] J. Sadeghi and B. Pourhassan, "Jet-quenching of the rotating heavy meson in a N=4 SYM plasma in presence of a constant electric field" Int. J. Theor. Phys. 50 (2011) 2305 arXiv:1001.0706 [hep-th]]

[39] K. B. Fadafan, B. Pourhassan and J. Sadeghi, "Calculating the jet-quenching parameter in STU background", Eur. Phys. J. C 71 (2011) 1785 [arXiv:1005.1368 [hep-th]]

[40] B. Pourhassan and J. Sadeghi "STU/QCD correspondence", Can. J. Phys. (2013) arXiv:1205.4254 [hep-th]] 